\title{
De la rhétorique de la qualité aux critères de la légitimité Le cas de l'école primaire genevoise, 2005-2015
}

\section{Olivier Maulini, Andreea Capitanescu Benetti, Julien Clénin, Danièle Périsset, Olivier Perrenoud, Laetitia Progin, Roberta Smania Marques et Carole Veuthey}

Entre 2005 et 2015, l'école primaire genevoise a connu une période d'innovations structurelles présentée comme une "réorganisation" destinée à "améliorer la qualité de l'enseignement»: directions d'établissements, projets d'établissements, conseils d'établissements, réseau d'enseignement prioritaire. L'analyse des textes officiels et leur confrontation à l'évolution observable de ces changements fait émerger quatre critères effectifs de légitimation de l'action publique en matière d'éducation: le fait d'assurer la paix sociale, de satisfaire les enseignants, de convaincre politiquement, de transcender les discussions. Ces résultats montrent que les vertus symboliques de chaque mesure sont mises à l'épreuve d'une rationalité politique qui semble devoir, non seulement donner satisfaction à des intérêts sectoriels, mais aussi composer entre leurs attentes contradictoires.

«Viser la qualité de l'enseignement tout au long du parcours de formation des élèves». "Assurer la qualité dans les classes et les écoles». "Attirer, former et retenir des enseignants de qualité». "Améliorer la qualité des études et de la formation de [ces] enseignants». «Former [les directions d'école] à un management stratégique orienté vers le développement de la qualité». "Assurer la qualité des différentes composantes de leur formation» (Maulini et al., 2015). Dans le langage des instances cantonales, nationales ou internationales en charge de l'éducation, la qualité d'une formation semble souvent nécessaire et suffisante pour fonder sa légitimité (Behrens, 2007; Daschner \& Rolff, 2011). Peut-être parce que l'idée de qualité fait l'unanimité. Aussi parce que la définir comme «la relation entre les prestations du système et la satisfaction des clients» (Gather Thurler, 1998) évoque moins un critère d'évaluation discutable que le verdict (forcément positif) d'une satisfaction incontestable parce que subjective (Boltanski \& Chiapello, 1999; Gramaccia, 2008).

Mais pour les sciences politiques, la «légitimité démocratique» (Rosanvallon, 2008) est forcément conflictuelle. Elle est basée sur des transactions qui supposent certes une communication plus ou moins stratégique, une rhétorique et un maniement de symboles capables de produire «a semblance of reality» 
(Edelman, 1964, p. 190), mais sans que l'agir dramaturgique et son principe régulateur de "présentation de soi» (Goffman, 1973a,b) ne puissent prétendre au dernier mot. D'un côté, il est clair qu'un élu du peuple n’inspire confiance que s'il «cherche à entretenir l'impression selon laquelle il vit conformément aux nombreuses normes qui servent à l'évaluer» (p. 237). D'un autre côté, les "effets d'annonce» ou les "tours de passe-passe» font justement partie de ce qu'il est politiquement dangereux de se voir reprocher. Notre intention n'est donc pas ici de dénoncer naïvement l'écart aisément explicable entre les discours et les actes, mais d'étudier comment les symboles sont mis à l'épreuve de la réalité, et ce que ce phénomène peut éventuellement nous dire des critères effectifs de légitimité (Maulini, 2009). Nous le ferons dans le contexte récent de l'école primaire genevoise et de la crise de confiance qu'elle subit en période d'instabilité économique et morale. Les questions suivantes orienteront notre analyse: de quelles innovations observables la rhétorique de la qualité s'est-elle accompagnée localement? Quels sont les critères de légitimité qui font que ces innovations ont eu tendance à perdurer ou non? Quelles tensions observe-t-on, cas échéant, entre la manipulation des symboles et les contraintes du réel, avec quelles conséquences possibles pour l'école et la valeur de son travail, in fine?

D'un point de vue méthodologique, nous avons réuni un corpus exhaustif des textes programmatiques et des directives couvrant la période 2005-2015². Par induction croisée des régularités et des variations (Maulini et al., 2012), nous avons catégorisé ces données en le confrontant à une observation documentée de l'évolution des pratiques réelles, pour répondre tour à tour à nos trois questions. Dans le texte qui suit, nous commençons par présenter (1) le contexte de la recherche, puis nous identifions (2) les innovations localement implémentées et (3) leurs critères de légitimité à la lumière de leur évolution récente, avant de conclure en discutant (4) des tensions observables entre la logique consistant à assurer la qualité des prestations ou à rassurer l'opinion publique à leur propos.

\section{Contexte agité, stratégie de (ré)conciliation}

En contexte de compétition mondialisée, les démocraties et leurs systèmes éducatifs font face à des attentes sociales pressantes et angoissées. Pour Ravitch (2010, p. 16), "what once was an effort to improve the quality of education turned into an accounting strategy; measure, then punish or reward; (...) the strategy produced fear and obedience among educators». La Suisse ne fait pas exception à cette règle. Selon Forster (2008, p. 108-109), le «malaise» et le «désarroi» dominent dans les cantons-villes qui concentrent l'essentiel de la précarité, de la fragmentation et de la compétition sociale perceptibles dans le pays. En réponse à ces contraintes, Genève a lancé une réforme visant à réduire l'échec scolaire à la fin du $20^{\mathrm{e}}$ siècle, réforme controversée et finalement rejetée par votation populaire en 2006 au profit d'une sélection par le redoublement et 
les moyennes chiffrées (Hutmacher, 1993; Crahay, 2010). Les enquêtes PISA venaient, il est vrai, d'envenimer la situation en classant le canton au dernier rang de la Confédération.

En écho à ces événements et par souci de stabiliser l'école après une décennie d'innovations et de polémiques à leur propos, le ministre en charge de l'instruction publique a défini, dès 2005, treize priorités pour l'instruction publique. Au temps de la créativité et de la controverse devait succéder celui du contrôle et de la pacification. "Ces treize priorités s'inscrivent dans une logique de recadrage de l'action publique, d'évaluation et de compte rendu, basée sur des indicateurs, et dans la recherche d'un nouveau souffle en matière de concertation» (Département de l'instruction publique [DIP], 2005, p. 3). Allant de "combattre l'échec scolaire» à «lutter contre la maltraitance» ou «intensifier l'apprentissage du français», les mesures étaient elles-mêmes encadrées par deux items ouvrant et concluant la liste des engagements: «Renforcer la cohérence et la qualité du système scolaire» (priorité 1) et «Une politique fondée sur l'évaluation et la participation» (priorité 13).

L'intention générale de (ré)conciliation s'est ensuite déclinée en une série d'annonces énumérant les instruments de cadrage et de contrôle censés assurer sa mise en œuvre. Par convention (Conférence intercantonale de l'instruction publique de la Suisse romande et du Tessin [CIIP], 2007), les cantons romands s'étaient dans l'intervalle dotés d'un plan d'études et d'épreuves communes pour l'enseignement obligatoire compatibles avec les standards nationaux du concordat HarmoS, lui-même issu de la révision constitutionnelle approuvée par $85 \%$ du peuple suisse et stipulant que «la Confédération et les cantons veillent ensemble à la qualité et à la perméabilité de l'espace suisse de formation» (Conférence suisse des directeurs cantonaux de l'instruction publique [CDIP], 2007). Pour compléter ce dispositif, un nouveau fonctionnement a été mis en place en 2008 au degré primaire genevois, peu avant la fusion de cette entité avec le degré secondaire I sous la bannière d'une Direction générale de l'enseignement obligatoire. Présentée sous le titre «L'école primaire genevoise se réorganise pour améliorer la qualité de l'enseignement", cette évolution ne devait pas passer pour une nouvelle réforme, mais compiler quatre changements sectoriels:

1. L'instauration de directions d'établissements scolaires chargées de superviser les apprentissages des élèves et le travail des enseignants.

2. La généralisation de projets d'établissement mettant au centre de leurs priorités la définition d'objectifs, d'indicateurs de réussite, de procédures d'évaluation et de régulation par les résultats.

3. La création de conseils d'établissement chargés de renforcer les liens, le dialogue et la confiance entre les familles et école.

4. La consolidation et l'extension d'un réseau d'enseignement prioritaire réservé aux quartiers socialement défavorisés, et se fixant des buts spécifiques en échange de ressources humaines et financières renforcés. 
Cet ensemble d'initiatives combinait plusieurs modes de régulation: les résultats mesurés; les directives institutionnelles; la participation des usagers; l'autonomie concédée aux équipes et aux établissements; le pouvoir délégué au directeur d'associer les enseignants aux décisions ou de s'assurer de la conformité de leurs pratiques. Le tout était chapeauté d'un Observatoire du fonctionnement de l'enseignement primaire, instance indépendante réunissant des experts de plusieurs institutions de recherche et de formation, mais extérieure à la liste des quatre leviers de transformation. Notre analyse va porter sur les modes de régulation opérés par l'ensemble du dispositif, pour voir ce qu'il a régulé mais aussi ce qui l'a régulé lui-même, donc ce qui a assuré ou non sa pérennité et son développement.

\section{Un contrôle renforcé? Quatre innovations}

Avant de voir comment les choses ont évolué, présentons les quatre innovations sectorielles, en prenant appui sur les documents officiels explicitant leur rôle et justifiant leur apparition. Nous le ferons dans l'ordre adopté par le Département de l'instruction publique lui-même, en passant des directions aux projets puis aux conseils d'établissement, pour terminer par le réseau d'enseignement prioritaire créé juste auparavant.

\section{Les directions d'établissement}

L'un des instruments le plus visible dans le nouveau fonctionnement a été la création d'un statut nouveau, remplaçant les anciens inspecteurs itinérants: celui de directrice ou directeur d'un établissement. Le but déclaré de ce changement était de favoriser une autonomie centrée sur les besoins du contexte local et de privilégier un encadrement de proximité, dont une recherche avait récemment regretté la fragilité (Papart, 2003). Chaque école se dotait ainsi d'un profil propre afin de coordonner la vie scolaire en interaction avec les partenaires éducatifs. L'introduction du rôle de directeur était en outre censée favoriser une certaine cohésion entre les écoles primaires et secondaires, qui ont désormais toutes à leur tête une direction d'établissement.

Quel a été l'impact de cette innovation? Selon l'Observatoire de l'enseignement primaire [OFEP] (OFEP, 2010) l'introduction d'un soutien hiérarchique de proximité a été propice à la cohérence des établissements en milieu dit «sensible». On constate $75 \%$ ou plus d'opinions favorables à l'introduction des directeurs d'établissement chez les enseignants, notamment en lien avec la manière dont le directeur représente l'établissement auprès des partenaires externes, dont il peut résoudre rapidement les problèmes courants, et dont il assume un rôle de soutien envers ses collaborateurs. Malgré ce résultat, on peut relever que l'introduction des directeurs s'est déroulée sous le regard méfiant d'une majorité d'enseignants craignant l'arrivée de "petits chefs" à la porte de 
leur classe, et/ou se demandant si la fonction ne pourrait pas se limiter aux écoles du réseau d'enseignement prioritaire et à leurs besoins particuliers d'autorité de proximité (Service de recherche en éducation [SRED], 2012). Le syndicat lui-même a soutenu l'innovation du bout des lèvres, sans négliger de dénoncer ouvertement chaque abus de pouvoir éventuel (Progin, 2014).

Dans ce contexte, le nombre de directions a diminué progressivement. En octobre 2015, elles étaient 58, contre 93 à leur création en mai 2008 (DIP, 2016). Cette tendance s'est accélérée sous l'effet d'une motion parlementaire demandant la réduction de la charge financière et l'assignation d'une obligation d'enseigner à chaque directeur. En passant, les directeurs ont embrassé la responsabilité d'un établissement plus grand et multi-sites, s'approchant de facto de la condition des précédents inspecteurs de circonscription. La fonction hiérarchique de proximité est ainsi plus ou moins clairement affaiblie. Le Département de l'instruction publique doit s'accommoder de ce reflux, et il y est d'autant plus contraint que les acteurs de terrain ne se battent guère pour défendre une ressource apparemment moins précieuse pour eux que les enseignants chargés du soutien pédagogique, les maîtres de discipline artistique et sportive, ou le ratio d'élèves par classe.

\section{Les projets d'établissement}

Autant l'arrivée des directeurs a-t-elle introduit une rupture, autant les projets d'établissement se sont-ils inscrits dans la continuité des réformes précédentes, y compris celle que la votation populaire de 2006 venait apparemment de désavouer. Rétablissant contre son gré les modalités de sélection, le Département de l'instruction publique a en effet cherché à concilier un pilotage par le centre censé apaiser les esprits et une délégation de responsabilité gage d'autonomie et d'engagement des équipes enseignantes dans les quartiers.

Par l'obligation formelle de rédiger et de conduire un projet, l'établissement scolaire devient autre chose qu'une simple division administrative placée sous le contrôle d'un directeur. Officiellement, il accède au rang d'unité de travail et d'analyse de ce travail, de communauté professionnelle solidairement comptable et tributaire de ses relations avec ses partenaires (élèves, parents, élus, autres professionnels de l'éducation, de l'animation, de la santé, des services administratifs et techniques, etc.). Selon les documents officiels (DIP, 2005), l'introduction du projet d'établissement va de pair avec son autonomie, un partenariat avec les familles, une politique fondée sur l'évaluation, l'auto-évaluation et la participation. Pour cela, des analyses et des feedbacks externes sont assumés par l'institution, sur la base des bilans rédigés par les écoles (Gros, Guilley, Jaeggi \& Sermet, 2012).

La taille des établissements et le nombre de sites qu'ils regroupent ayant quasiment doublé depuis leur création, il est devenu de plus en plus difficile de conduire des projets à la fois rassembleurs et tenant compte des spécificités et des problématiques locales. La planification des projets arrive en ce moment 
à la fin d'un cycle de quatre ans, et le nouveau dispositif paraît évoluer vers des contraintes moins fortes, des initiatives coordonnées plus modestes, davantage inspirées de la classique pédagogie de projet (des activités formatrices impliquant les élèves à l'échelle d'une école) que du nouveau management public (des objectifs mesurables à atteindre par un collectif d'enseignants). Certains acteurs regrettent ce reflux, en le mettant sur le compte d'une bureaucratisation progressive de leur idéal initial d'autogestion. La majorité paraît s'en accommoder voire s'en féliciter, comme si le sentiment d'un simulacre de participation débouchait sur un surcroît de repli.

\section{Les conseils d'établissement}

Si l'établissement a émergé comme une personne morale capable de prendre des initiatives et des décisions, c'est en particulier sous la pression, voire à la demande des parents. Une politique officiellement basée sur la concertation pouvant difficilement ignorer cet échelon, la création des conseils d'établissement a voulu afficher une volonté de collaborer avec les familles, dans le prolongement, là aussi, des réformes précédentes. Pour le ministre à l'origine de sa création, cet espace local de concertation devait certes offrir un espace d'information et de débat aux usagers, mais avec un impact (fût-il indirect) sur le fonctionnement régulier de l'établissement, les pratiques pédagogiques et les apprentissages des élèves. "Lieux d'information, de consultation, de proposition et de délibération, les conseils d'établissement renforcent la démocratie et la participation. En créant ces conseils, j'ai voulu développer les liens entre l'école, la famille et les communes afin de créer un climat propice à l'apprentissage des élèves» (Parti socialiste genevois [PSG], 2013). La démocratie participative était promue comme une valeur en soi, d'autant mieux qu'elle contenait la promesse d'une efficacité grandissante du travail scolaire.

Le règlement sur les conseils (DIP, 2007) a prévu leur élection tous les quatre ans et leur présidence ex officio par le directeur d'établissement. Ces cénacles sont «des lieux de d'information, de consultation, de proposition et de décision». Les enseignants restent maîtres de la pédagogie, mais le conseil «est informé notamment des questions relatives aux plans d'études, aux programmes scolaires et aux moyens d'enseignement ainsi qu'aux conditions d'admission, d'orientation et de promotion des élèves». Les usagers et les partenaires exercent ainsi un contrôle partiel sur les pratiques. Ils peuvent en outre «décider des actions et des mesures à intégrer dans le projet d'établissement, permettant de contribuer de la manière la plus appropriée à développer un climat propice à l'apprentissage des élèves». Cette formule revient avec insistance, sans doute pour signifier que la qualité de l'enseignement dépend des compétences des enseignants mais aussi du climat alentours et d'une solidarité élargie à la communauté éducative.

De son côté, l'Observatoire conclut que «le conseil d'établissement favorise l'expression de tous les partenaires" selon $61 \%$ des enseignants, $70 \%$ des directeurs et jusqu'à $82 \%$ des parents (OFEP, 2010a). Cependant, «le conseil d'éta- 
blissement doit encore s'affirmer», «il accroît la transparence de l'établissement», mais «il n'incarne pas son autonomie» selon deux tiers des enseignants et des directeurs. Quelque temps plus tard, un rapport de synthèse relève que «le conseil d'établissement n'est pas totalement perçu comme une instance de régulation de l'autonomie, ni comme répondant aux besoins spécifiques de la population» (OFEP, 2010b, p. 17). Il n'est logiquement valorisé que lorsque les discussions et les débats débouchent sur des projets tangibles, ce qui n'est pas la règle selon les acteurs (Jaeggi \& Schwob, 2010). Ceci explique peut-être la relative désuétude vers laquelle l'innovation a finalement évolué. Depuis la rentrée 2015, les conseils ne semblent plus actifs que dans les établissements qui tiennent à leur pérennité, sans que le Département ne leur demande de rendre des comptes comme auparavant. La satisfaction des acteurs locaux pourrait davantage peser que la volonté politique dans leur prolongation.

\section{Le réseau d'enseignement prioritaire}

Le Département de l'Instruction Publique genevois a créé son réseau d'enseignement prioritaire à la rentrée 2006. Pris dans la tourmente héritée de la réforme précédente, le ministre de l'éducation a ainsi voulu reprendre l'initiative sur le front de la lutte contre l'échec scolaire, en concentrant ses efforts en direction des quartiers sociologiquement et économiquement défavorisés. Indépendamment de son efficacité potentielle, ce pas de côté a eu la vertu de déplacer le débat et même de le neutraliser partiellement, en laissant de facto les milieux favorisés à l'écart des innovations.

Les mesures mises en place au sein du réseau se sont caractérisées par une augmentation des ressources humaines, un encadrement plus généreux des élèves, un soutien au travail socio-éducatif, des échanges facilités entre établissements et avec les partenaires du réseau. La création d'un poste d'éducateur par établissement a été particulièrement plébiscitée par les enseignants, que ce nouveau protagoniste est venu soulager de tâches jugées lourdes et incompatibles avec le «vrai travail» d'instruction: circuler dans l'école et ses environs, dialoguer informellement avec les élèves, rencontrer et conseiller leurs familles, les soutenir dans leurs démarches administratives, etc.

Dans ces établissements, différentes modalités de travail ont intensifié la coopération entre enseignants et avec des réseaux interprofessionnels autour des élèves en difficulté, plus précisément dans le domaine de la lecture et de l'entrée dans l'écrit (SRED, 2012). Mais malgré les dispositifs centrés sur des renforcements didactiques et l'organisation du travail scolaire, les progrès des élèves sont restés modestes par rapport aux évolutions observables ailleurs dans le système (Soussi \& Nidegger, 2015). Les auteurs des différents rapports sont restés prudents, rappelant régulièrement que plusieurs années d'expérimentation sont souvent nécessaires avant d'observer d'éventuels sauts qualitatifs. Sans surprise, on a par exemple constaté que diminuer le nombre d'élèves par classe, associer des éducateurs aux enseignants et travailler davantage en équipe étaient 
des composantes importantes mais pas suffisantes pour endiguer les difficultés liées à l'origine sociale des élèves. Cela n'a pas empêché le dispositif de s'installer et d'être considéré comme le nouveau signe tangible de la volonté genevoise de réduire les inégalités.

\section{Régulations observables et critères de légitimité}

Entre 2005 et 2015, les quatre innovations initiales ont donc connu de sorts en partie communs, en partie singuliers. Peu ont prospéré, certaines semblent aujourd'hui menacées de mort lente. On peut considérer que des régulations se sont opérées en dix ans, et qu'elles ont pu être accélérées par le changement de ministre intervenu récemment. Nous allons partir du principe que ce qui gouverne réellement l'école est un faisceau de critères de légitimité qui ne sont moralement ni bon ni mauvais, mais qui se donnent à voir dans la manière dont une collectivité conserve, modifie ou abandonne effectivement des pratiques.

\section{Assurer la paix sociale}

À Genève comme dans beaucoup d'autres contextes politiques, deux critères sont le plus souvent invoqués pour justifier les réformes ou les contre-réformes éducatives. Le premier est celui de la prospérité économique: mieux les enfants sont formés, mieux ils trouvent à s'intégrer dans le monde du travail, et mieux la collectivité défend ses intérêts dans une compétition globalisée. Le second critère est celui de la justice sociale: moins les savoirs sont également répartis dans une société, plus la partie qualifiée de la population peut imposer ses vues à celle qui doit la croire sur parole. C'est sans doute pour ces deux raisons combinées - l'une plutôt de droite, l'autre plutôt de gauche - que la loi genevoise sur l'instruction publique stipule que l'enseignement doit «tendre à corriger» les inégalités de réussite scolaire des élèves.

Dans les faits, le critère du lien social et celui de l'intérêt collectif peuvent entrer en conflit. Forts de leur autonomie supposée, certains établissements genevois ont par exemple cherché à mieux intégrer les élèves en difficulté ou d'origine étrangère dans leurs classes ordinaires. Les structures qui étaient réservées à ce public se sont ouvertes, afin d'immerger chaque enfant dans un milieu hétérogène, jugé pédagogiquement plus stimulant. Dans ces écoles, des parents de milieu socio-culturellement dominant ont pu s'élever contre ces pratiques en invoquant le danger d'un nivellement par le bas. Les équipes et les directions à l'origine des dispositifs innovants se sont trouvées prises de court, embarrassées face à une résistance qu'elles désapprouvaient idéologiquement, mais qui mettait en danger la paix sociale, la légitimité de l'école et, par ricochet, le statut et les conditions de travail des élèves défavorisés eux-mêmes. Pour «calmer le jeu» et restaurer la tranquillité perdue, des structures étanches ont 
été par endroits restaurées. Ailleurs, l'anticipation de ce risque a pu dissuader (consciemment ou inconsciemment) les autres établissements de se distinguer. Dans un contexte général de défiance vis-à-vis de l'école, chaque conflit isolé peut vite intéresser la presse locale et se déplacer jusqu'au pouvoir central. Au-delà des promesses d'autonomie, d'équité et d'efficacité incluses dans la promotion de la qualité, la realpolitik peut donc imposer sa loi de facto: l'ordre social se maintient parfois moins par la recherche à long terme de justice et/ou de prospérité, que de garantie à court terme d'un minimum de consensus, fût-ce aux dépens d'une minorité silencieuse et marginalisée.

\section{Satisfaire les enseignants}

Pacifier au dehors donc, mais aussi au-dedans. Nous avons vu, par exemple, que le réseau d'enseignement prioritaire, ses directions d'établissement réactives et ses éducateurs mobiles pouvaient décharger les enseignants des tâches de médiation que beaucoup d'entre eux jugent étrangères à leur tâche d'instruction. A contrario, les projets et les conseils d'établissement ont pu être perçus à maints endroits comme des obligations formelles, voire comme des contraintes bureaucratiques peut-être utiles à l'autorité scolaire pour afficher son parti pris de responsabilisation des acteurs, mais au prix d'une réduction indirecte de l'autonomie de chacun. Force est de constater que certaines mesures ont donc satisfait la profession plus que d'autres, ce qui leur a donné davantage de chances de durer au-delà du ministre les ayant personnellement instaurées.

Les enseignants ne décident pas de tout dans l'école, loin de là. Nous venons de voir que des relations apaisées avec les usagers étaient elles aussi (et souvent d'abord) un critère objectif de régulation, quitte à ce que les professionnels et leurs manières d'envisager leur rôle fassent partiellement les frais d'un tel ajustement. On voit surtout combien la situation est complexe, puisque l'accord des maittres est certes une composante de la bonne entente générale, mais aussi son principal obstacle potentiel lorsque la rationalité professionnelle s'oppose à la rationalité politique (Gather Thurler \& Perrenoud, 1991). Dans le contexte genevois, on observe que chacune de ces rationalités est limitée, c'est-à-dire qu'elle vise moins un équilibre optimal qu'une solution à peu près satisfaisante, mais qu'elle est aussi ambiguë, puisque la satisfaction des uns est à la fois contraire et en partie nécessaire à la satisfaction des autres. Si nous considérons l'assentiment des enseignants comme un critère autonome de légitimation, c'est parce que les faits observés ont montré comment ce régulateur peut entrer en conflit avec les attentes de leur hiérarchie et/ou celles de leur environnement.

\section{Convaincre politiquement}

Un arbitrage est toujours possible par l'autorité de la loi et de la décision politique. Le pouvoir exécutif est normalement en charge de la gestion de l'État, le législatif de fixer les conditions-cadres d'un bon gouvernement. Mais en contexte de crise de confiance, d'impatience croissante et d'instabilité des majorités, le pilotage de 
l'action publique peut se voir soumis à des tensions externes et internes grandissantes. Dans le cas de l'école genevoise, le parlement ou le peuple lui-même ont récemment déterminé les manières d'évaluer les apprentissages des élèves, d'organiser les filières des écoles, de découper l'horaire de travail hebdomadaire, de définir les charges des directeurs, de calibrer la formation des enseignants, etc., toutes questions qui était jadis dévolues au règlement. Qu'ils le regrettent ou qu'ils s'en félicitent, les professionnels ont appris à vivre dans un monde où ce qu'ils ne parviennent pas à arbitrer entre eux (au niveau exécutif) est désormais externalisé par une minorité (au niveau législatif).

Mais passer d'un plan à l'autre change aussi les critères de jugement. Ceux des initiés (enseignants, cadres, experts) peuvent s'ancrer dans leur cadre théorique et déontologique, ceux des profanes (élus, médias, population) dans des représentations et des normes les prenant à revers. La préférence pour l'austérité financière et la discrétion hiérarchique (deux vertus helvétiques) a par exemple réduit de moitié le nombre des directeurs d'établissement, tout en leur assignant une charge d'enseignement. L'autonomie locale initialement valorisée ne s'est pas imposée comme un profit évident et politiquement soutenable par une majorité de circonstance. Par la diminution des effectifs et des prérogatives des directeurs, l'institution semble admettre que le contrôle de proximité n'apporte pas de gain d'efficacité, en tout cas pas un gain capable de compenser le coût des postes engagés. Ce contrôle de proximité a souvent été considéré par ses détracteurs comme inadéquat, indépendamment de ses effets mesurables, précisément longs et difficiles à mesurer. Mais le temps politique n'est pas celui de la recherche, et une innovation doit convaincre immédiatement avant d'avoir pu faire ses preuves ou non. La légitimité sociale des cadres intermédiaires a donc été rapidement remise en question, dans un contexte culturel et politique peu favorable à la verticale de l'autorité, et où le pouvoir et le contrôle sont souvent euphémisés au nom de l'égalité entre pairs.

\section{Transcender les discussions}

Les usagers, les professionnels, les élus et l'opinion publique sont donc tous à convaincre ou à séduire. Directement ou indirectement (par exemple à travers la presse ou les réseaux sociaux), c'est avec eux qu'il faut discuter; et quand la discussion menace de trop durer, c'est en se tournant vers l'extérieur du canton qu'il faut trouver le moyen de la transcender. Dans l'idéal du chercheur (et peut-être de l'instituteur), des faits et des savoirs attestés devraient souvent suffire à arbitrer les différends, à distinguer le vrai du faux. Le cas genevois montre plutôt (1) qu'il est difficile de dépassionner les débats sur l'école et de les trancher (même partiellement) au moyen d'arguments scientifiques (Mottier Lopez, 2014), (2) qu'il est plus économique de passer par des règles, certes elles aussi discutables mais définies ailleurs, dans un espace de normalisation à la fois distant de l'arène locale et moins remuant qu'elle (Maulini, 2008). En Suisse, c'est particulièrement le cas d'une coordination intercantonale non seulement 
fondée sur le principe de la concordance, mais aussi partiellement soustraite au contrôle (et à l'agitation) des parlements locaux (Chollet, 2011).

La rhétorique de la qualité affirme subordonner la validation des moyens aux résultats qu'ils permettent ou non d'atteindre. La réalité est plus ambiguë: plus les performances attendues sont longues à obtenir et incertaines à définir (comme c'est le cas en éducation), plus le débat peut se focaliser sur les vertus - peut-être apparentes mais au moins immédiates - des procédures censées les produire. C'est précisément au niveau régional que les cantons romands ont développé une batterie d'instruments de travail conçus par des experts et validés par les ministères: convention scolaire romande, déclaration commune sur les finalités de l'école publique, plan d'études romand pour les trois cycles de l'école obligatoire, grille-horaire de référence, moyens d'enseignement pour tous les degrés et toutes les disciplines scolaires, épreuves standardisées en préparation. Toute initiative cantonale doit s'inscrire dans ce cadre sous peine de menacer le concordat tout entier. Autant dire que les marges de manœuvre se réduisent, pour le meilleur ou pour le pire selon chaque partenaire impliqué. Les cantons ruraux peuvent par exemple craindre l'instabilité des centres urbains; ceux-ci envier le calme et la sérénité des vallées périphériques. Fait significatif: une seule question ne doit, pour l'instant, pas être posée en commun; c'est celle des modalités de classement des élèves, trop sensible pour être partagée entre les cantons.

\section{Conclusion: assurer ou rassurer?}

Résumons nos résultats: la paix sociale, la satisfaction des enseignants, l'adhésion du pouvoir politique et la conformité à des règles transcendantes apparaissent comme les quatre critères de régulation principalement à l'œuvre dans l'évolution de l'école primaire genevoise. Quelle que soit la réforme envisagée et ses lettres de noblesse, elle risque d'échouer sans respecter ces conditions. Ce constat est peut-être en partie propre au contexte suisse et à son modèle fédéraliste et référendaire de décision, mais on peut en imaginer des variantes dans d'autres démocraties. Où que l'on soit, peu importe la pertinence intrinsèque d'une proposition d'action: l'important est qu'elle fasse, sinon l'unanimité, au moins la concorde entre les milieux les plus à même de valider ou au moins de tolérer des projets et des idées (Périsset, 2015). La ministre genevoise actuellement en charge de l'instruction publique a ainsi fait sienne la cause de «l'école inclusive». Elle peut compter sur le soutien des milieux actifs dans la défense des enfants en situation de handicap ou touchés par différents "troubles de l'apprentissage» bien identifiés. Elle bute en même temps sur les objections des enseignants qui lui demandent non seulement des moyens à la hauteur de cette intention, mais aussi moins de contradiction structurelle entre sélection précoce et inclusion facilitée. Comme à toute table de négociation, la légitimité d'un accord dépend des rapports de forces entre les signataires impliqués. La «satisfaction du client» 
est un critère marchand, supposant un interlocuteur autosuffisant.

En situation de transaction politique, le pouvoir organisateur garde moins le cap parce qu'il assure la séduction d'un consommateur (comme le ferait un commerçant), que parce qu'il rassure les parties impliquées dans une confrontation (en leur donnant des signes extérieurs d'équilibre et d'impartialité). "Ce qui préoccupe les individus [fussent-ils diplomates, ministres ou enseignants], c'est moins la question morale de l'actualisation des normes, que la question amorale de la mise au point d'une impression propre à faire croire qu'ils sont en train d'actualiser ces normes» (Goffman, 1973a, p. 237; souligné par nous). Se présenter sous un jour favorable peut-être moralement dénoncé comme un réflexe opportuniste, mais ce procès d'intention fait long feu si le pouvoir à l'œuvre rétorque qu'il agit ainsi au nom d'un intérêt, non pas égoïste, mais supérieur à tous les autres. Après une période de fièvre et d'affrontement dans et autour de l'école genevoise, comment s'étonner de l'émergence d'un champ lexical («recadrage, cohérence, qualité, concertation») valorisant, en retour, l'entente cordiale et la stabilité?

Nous l'avons dit d'emblée: l'efficacité symbolique n'exclut pas l'efficacité instrumentale; elle complique le jeu. Un gouvernement peut à la fois communiquer et agir efficacement: à la limite, sa communication fait partie de son action. Ne nous méprenons donc pas sur l'usage des symboles, des idées et des mots dans l'orientation et la régulation des politiques scolaires. Autant que le reste, ils font partie des ressources mobilisables pour gouverner, innover et obtenir des résultats. Mais s'ils cherchent à séduire, c'est moins pour «satisfaire» une clientèle unilatéralement calculatrice que pour composer entre des groupes dont les valorisations peuvent varier, mutuellement se contester, et finalement produire une demande collective de modération des attentes sectorielles. Le destin des réformes est donc probablement de subir l'usure des conflits autant que celle du temps. De là, une nouvelle recherche pourrait se demander comment les enseignants vivent et perçoivent la légitimation de l'action publique dans le champ de l'éducation. Eux dont le métier est de manipuler des symboles, comment jugent-ils ce qu'en fait l'institution?

\section{Références bibliographiques}

Behrens, M. (Éd.). (2007). La qualité en éducation: pour réfléchir à la formation de demain. Québec: Presses de l'Université du Québec.

Boltanski, L. \& Chiapello, E. (1999). Le nouvel esprit du capitalisme. Paris: Gallimard.

Conférence suisse des directeurs cantonaux de l'instruction publique [CDIP] (2007). Accord intercantonalsur l'harmonisation de la scolarité obligatoire (concordat HarmoS). Consulté le 15 avril 2015 dans: http://edudoc.ch/record/24710/files/HarmoS_f.pdf

Conférence intercantonale de l'instruction publique de la Suisse romande et du Tessin [CIIP] (2007). Convention scolaire romande. Consulté le 15 avril 2015 dans: http://www.ciip.ch/ documents/showFile.asp?ID=2176

Crahay, M. (2010). Les réformes pédagogiques échouent, et pourtant l'école change! In P. Giliéron Giroud \& L. Ntamalikiro (Éd.), Réformer l'évaluation scolaire: mission impossible? (pp. 231-264). Berne: Peter Lang. 
Chollet, A. (2011). Défendre la démocratie directe. Sur quelques arguments antidémocratiques des élites suisses. Lausanne: Presses polytechniques et universitaires romandes.

Daschner, P. \& Rolff, H.-G. (2011). Qualitätsmanagement. Journal für Schulentwicklung, 1, 4-5.

Département de l'instruction publique [DIP] (2005). 13 priorités pour l'instruction publique. Genève: DIP. Consulté le 15 avril 2015: ftp://ftp.geneve.ch/dip/cp13priorites200105.pdf

Département de l'instruction publique [DIP] (2007), Règlement sur les conseils d'établissement C1 10.19 entrée en vigueur le 29 décembre 2007. Consulté en ligne le $1^{\mathrm{er}}$ avril 2015 dans: https://www.ge.ch/legislation/rsg/f/s/rsg_C1_10P19.html

DIP (2016) direction générale de l'enseignement primaire. Genève: DIP. Csonulté de 26 août 2016: www.ge.ch/primaire

Edelman, M. (1964). The symbolic Uses of Politics. Urbana \& Chicago: University of Illinois Press.

Forster, S. (2008). L'école et ses réformes. Lausanne: Presses polytechniques et universitaires romandes.

Gather Thurler, M. (1998). Manager, développer ou évaluer la qualité de l'école? In G. Pelletier et R. Charron (Éd.), L'évaluation institutionnelle de l'éducation: défi, ouverture et impasse (pp. 83-100). Québec: Éditions Afides.

Gather Thurler, M. \& Perrenoud, Ph. (1991). L'école apprend si elle s'en donne le droit, s'en croit capable et s'organise dans ce sens! In Société suisse de recherche en éducation (Éd.), L'institution scolaire, est-elle capable d'apprendre? (pp. 75-92). Luzern: Zentralschweizerischer Beratungsdienst für Schulfragen.

Goffman, E. (1973a). La mise en scène de la vie quotidienne. 1. La présentation de soi. Paris: Minuit.

Goffman, E. (1973b). La mise en scène de la vie quotidienne. 2. Les relations en public. Paris: Minuit.

Gramaccia, G. (2008). Qualité, projet, numérique: trois variations symboliques de l'efficacité gestionnaire. Médiation et Information, 29, 55-67.

Gros, D., Guilley, J.-M., Jaeggi, J.-M. \& Sermet, G. (2012). Évaluation externe des projets d'établissement (EVALEX). Rapport de synthèse. Genève: Service de recherche en éducation.

Hutmacher, W. (1993). Quand la réalité résiste à la lutte contre l'échec scolaire (Cahier ${ }^{\circ} 36$ ). Genève: Service de la recherche sociologique.

Jaeggi, J.-M. \& Schwob, I. (2010). Les établissements du REP dans leur environnement. Quatrième rapport intermédiaire: quatre études de cas. Année scolaire 2008-2009. Genève: Service de recherche en éducation. Consulté le 1er avril 2015 dans: https://www.ge.ch/ recherche-education/doc/publications/docsred/2010/rep4.pdf

Maulini, O. (2008). Nouvelles compétences, anciennes méthodes? Mouvements et contre-mouvements dans l'école et la formation des enseignants: le cas de Genève et de la Suisse. Communication au colloque "La formation des enseignants en Europe: approche comparative» (Conférence des directeurs des Instituts universitaires de formation des maîtres, Paris, 9 et 10 décembre). Université de Genève, Faculté de psychologie et des sciences de l'éducation.

Maulini, O. (2009). Das öffentliche Bildungswesen und seine Legitimation: zwischen instrumenteller Vernunft und symbolischer Botschaft (L'école publique et sa légitimation: entre raison instrumentale et messages symboliques). Journal für Schulentwicklung, 2, 33-43.

Maulini, O., Capitanescu Benetti, A., Mugnier, C., Perrenoud, M., Progin, L., Veuthey, C. \& Vincent, V. (2012). Qu'est-ce qu'une «bonne pratique»? Raison pédagogique et rapport à l'efficacité chez les futurs enseignants. Questions Vives, 6(18), 36-56.

Maulini, O. et al. (2015). Assurer ou rassurer? De la rhétorique de la qualité aux pratiques de contrôle dans la formation des élèves, des enseignants et des directions d'établissement. Communication au Congrès «Qualitäts- und Bildungsdiskurs» de la Société suisse pour la recherche en éducation (PH Sankt-Gallen, 30 juin). Consulté le 1er décembre 2015 dans: www.unige.ch/fapse/SSE/teachers/maulini/publications.html 
Mottier Lopez, L. (2014). L'évaluation pédagogique va-t-elle enfin marcher sur ses deux pieds? Les enseignements de l'histoire récente de l'école primaire genevoise. Éducation et francophonie, 42(3), 85-101.

Observatoire de l'enseignement primaire [OFEP] (2010a). Première analyse des réponses apportées aux questionnaires enseignants, directeurs et parents. Genève: Département de l'instruction publique. Consulté le 1er avril 2015 dans: https://www.ge.ch/dip/doc/ actu/2010/100319_dossier_observatoire_primaire.pdf

Observatoire de l'enseignement primaire [OFEP] (2010b). Rapport du nouveau fonctionnement en 2009-2010. Première analyse de l'Observatoire du fonctionnement de l'enseignement primaire. Genève: Département de l'instruction publique. Consulté le $1^{\mathrm{er}}$ avril 2015 dans: https://www.geneve.ch/primaire/doc/observatoire/2009-2010/rapport_annuel_20092010_observatoire.pdf

Périsset, D. (2015). Mais pourquoi parle-t-on tellement de professionnalisation? Réflexion autour d'un concept polymorphe. In B. Wentzel, V. Lussi Borer \& R. Malet (Éd.), Professionnalisation de l'enseignement. Fondements et retraductions (pp. 17-40). Nancy: Presses universitaires de Nancy.

Ravitch, D. (2010). The Death and Life of the great american School System. How Testing and Choice are undermining Education. New York: Basic Books.

Papart, J.-P. (2003). La santé des enseignants et des éducateurs de l'enseignement primaire. Rapport à l'organisation du travail. Genève: Département de l'action sociale et de la santé.

Progin, L. (2014). Devenir chef d'établissement. Le désir de leadership à l'épreuve de la réalité. Enquête sur l'entrée dans un métier émergent. Université de Genève: Thèse de doctorat.

Parti socialiste genevois [PSG] (2013). Rapport d'activité du conseiller d'Etat Charles Beer devant le Congrès du Parti socialiste genevois. Samedi 9 mars 2013. Consulté le $1^{\mathrm{er}}$ avril 2015: http://www.ps-ge.ch/wp-content/uploads/2013/04/Rapport-du-Conseil-dEtat_2012.pdf

Rosanvallon, P. (2008). La légitimité démocratique. Impartialité, réflexivité, proximité. Paris: Seuil.

Soussi, A. \& Nidegger, Ch. (2015). Le réseau d'enseignement prioritaire genevois 8 ans après son introduction. Genève: Service de recherche en éducation. Consulté en ligne le $1^{\mathrm{er}}$ avril 2015 dans: https:/www.ge.ch/recherche-education/doc/publications/docsred/2015/ rep_8ans_apres_introduction.pdf

Service de recherche en éducation [SRED] (2012). Le suivi de la mise en place du Réseau d'enseignement primaire de 2006 à 2009 (Note d'information n 52). Genève: Département de l'instruction publique. Consulté le $1^{\text {er }}$ avril 2015 dans: https://www.ge.ch/ recherche-education/doc/publications/notesinfo/notes-sred-52.pdf

\section{Note}

1 Accès à ce corpus: http://www.unige.ch/fapse/SSE/teachers/maulini/publ-1601.pdf

Mots-clés: Politiques éducatives, innovation, contrôle, qualité, légitimité 


\section{Von der Qualitätsrhetorik hin zu Legitimitätskriterien. Das Fallbeispiel der Genfer Primarschule, 2005-2015}

\section{Zusammenfassung}

Zwischen 2005 und 2015 hat die Genfer Primarschule eine intensive Phase struktureller Innovationen durchlaufen. Im Namen der notwendigen «Reorganisation» zur «Verbesserung der Unterrichtsqualität» wurden Schulleitungen, Schulprojekte, Schulräte und ein Netzwerk von Schulen mit besonderen Bedürfnissen als Schwerpunkte der Schulentwicklung bestimmt. Der von uns unternommene Vergleich der vom Schulamt verfassten Texte mit den tatsächlich stattgefundenen Veränderungen im Schulalltag weist auf vier Kriterien der effektiven Handlungsfähigkeit im öffentlichen Schulfeld hin: unumgehbare Sicherung des Sozialfriedens, Zufriedenheit der Lehrpersonen, politische Überzeugungskraft, Fähigkeit, Worte in Taten umzusetzen. Unsere Forschungsergebnisse weisen darauf hin, dass die symbolischen Tugenden jeder Entwicklungsmaßnahme einem politischen Rationalitätstest unterstellt werden, dessen Sinn und Zweck nicht nur darin besteht, die Interessen aller Handlungspartner zufrieden zu stellen, sondern auch ihre widerstreitigen Erwartungen zu berücksichtigen.

Schlagworte: Bildungspolitik, Schulentwicklung, Kontrolle, Qualität, Legitimität

\section{Dalla retorica della qualità ai criteri di legittimità. II caso della scuola primaria ginevrina, 2005-2015}

\section{Riassunto}

Tra il 2005 e il 2015 la scuola primaria ginevrina ha attraversato un periodo di innovazioni strutturali, periodo presentato come una «riorganizzazione» volta a «migliorare la qualità dell'insegnamento»: direzioni di istituto, progetti di istituto, consigli di istituto, reti di insegnamento prioritario. L'analisi dei testi ufficiali e il loro confronto con l'evoluzione osservabile di tali cambiamenti fa emergere quattro criteri effettivi di legittimazione dell'azione pubblica in materia di educazione: il fatto di assicurare la pace sociale, di soddisfare gli insegnanti, di convincere politicamente, di trascendere le discussioni. Questi risultati mostrano che le virtù simboliche di ciascuna misura sono sottoposte al vaglio di una razionalità politica che sembra non solo dover dare soddisfazione a degli interessi di settore, ma altresì venire a patti con le loro aspettative contradditorie.

Parole chiave: Politiche educative, innovazione, controllo, qualità, legittimità 


\section{From rhetoric of quality to criteria of legitimacy: The case of the Geneva primary school, 2005-2015}

\section{Summary}

Between 2005 and 2015, the Geneva primary school system was involved in a period of structural innovation. This was presented as a 'reorganization' intended 'to improve the quality of teaching'. The reorganisation included placing principals in school to manage the establishments, school projects, boards of education and a network of priority education. Through analysis of the official texts and their impact within the evolution of these changes four criteria were brought to the foreground. This led to legitimization of public action regarding education: the fact to assure social stability, to satisfy teachers, to convince politically and to transcend the discussions. These results show that the symbolic virtues of every measure are tested by a political rationality which seems to owe, not only give satisfaction to sectorial interests, but also compose between their contradictory expectations.

Keywords: Education policies, innovation, control, quality, legitimacy 